\title{
CONSTRUCCIÓN DEL CONCEPTO DE ONDA Y DE FENÓMENOS ONDULATORIOS, EN ESTUDIANTES DE EDUCACIÓN MEDIA, UTILIZANDO UN MODELO VIRTUAL
}

\section{CONSTRUCTION OF THE CONCEPT OF WAVE AND WAVE PHENOMENA IN HIGHSCHOOL STUDENTS USING A VIRTUAL MODEL}

\author{
HERNANDO TAMAYO \\ Universidad Santiago de Cali \\ ALVARO PEREA \\ Universidad del Valle \\ WAYNER RIVERA \\ Universidad del Cauca
}

\section{RESUMEN}

Uno de los aspectos más llamativos es que al utilizar los métodos tradicionales en la enseñanza del concepto ondas se limita a dar conferencias, realizar prácticas experimentales y demostraciones, que proporcionan al alumno un aprendizaje mecánico, basado en la memorización y que no es significativa, debido a su complejidad. Los profesores representan las ondas por medio de diagramas en un tablero, eliminando su carácter dinámico. Cuando se producen las ondas mecánicas con un resorte helicoidal o en un balde, la naturaleza dinámica se demuestra, sin embargo, la dificultad de obtener mediciones precisas de los parámetros de ondas, como la amplitud, longitud de onda, la periodicidad, el cálculo de su frecuencia y la velocidad de propagación. La investigación sobre la complejidad de la construcción de estos conceptos nos lleva a diseñar una propuesta didáctica para ayudar al estudiante en la construcción científica del concepto de ondas. La propuesta tiene por objeto el uso de modelos virtuales de onda que permiten visualizar la naturaleza dinámica de las ondas, la medición de sus parámetros y la interacción del estudiante con el fenómeno para mostrar una relación de causa y efecto. Con este fin, dos plataformas (PhEt y el Proyecto Newton) fueron seleccionados, las cuales reproducen las ondas en un ambiente de micro-mundos digitales y modelos virtuales. La aplicación de la propuesta didáctica con un grupo de estudiantes de educación media dio excelentes resultados en la construcción del concepto de ondas y de los fenómenos ondulatorios. Las simulaciones permiten la visualización y comprensión de los modelos físicos de los fenómenos naturales, favoreciendo el aprendizaje en los procesos cognitivos de los estudiantes.

Palabras clave: modelo, plataforma, PhET, Proyecto Newton, ondas, función de onda, TICs 


\section{Abstract}

One of the most striking aspects is that, using traditional methods, teachingthe concept of waves is limited to delivering lectures and performingexperimental practices and demonstrations, which provide the student withlearning that is rote, mechanical, based on memorizing and rather notsignificant, on account of its complexity. Teachers represent waves by meansof diagrams on a blackboard, eliminating their dynamic nature. Whenproducing mechanical waves with a helicoidal spring or in a bucket withwaves, the dynamic nature is demonstrated; however, the difficulty ofobtaining precise measurements of the wave parameters such as amplitude,wavelength, periodicity, the calculation of its frequency and speed ofpropagation, is discovered. Research on the complexity of the construction ofthese concepts leads us to design a didactic proposal to help the student inthe scientific construction of the concept of waves. The proposal is designedusing virtual wave models that permit visualizing the dynamic nature ofwaves, the measurement of their parameters and the interaction of thestudent with the phenomenon by showing a cause and effect relationship.

For this purpose, two platforms (PhET and Newton Project) were selected, which reproduce the waves under an environment of digital micro-worldsand virtual models.

The application of the didactic proposal with a group of students in middleschool produced excellent results in the construction of the concept of wavesand of undulatory phenomena. The simulations allow for the visualization andunderstanding of the physical models of natural phenomena, favoringlearning in the students' cognitive processes.

Keywords: model, Platform, PhET, Newton Project, Waves, Wave function, TICs

\section{INTRODUCCION}

Usualmente, la enseñanza de la mayoría de los conceptos científicos $y$, en particular, en el campo de la Física, se encuentra limitado a clases magistrales, practicas de laboratorio y demostraciones que no aportan al estudiante un aprendizaje significativo debido al comportamiento dinámico que subyace en estos conceptos. Dentro de estos conceptos nos referimos al de onda y los fenómenos ondulatorios propios de este tema. Los profesores representan las ondas en el tablero por medio de diagramas estáticos no permitiendo mostrar su carácter dinámico. Al tratar de hacerlo produciendo ondas mecánicas con un resorte helicoidal o en una cubeta de ondas, se muestra su carácter dinámico pero se descubre la dificultad de obtener medidas precisas de parámetros de onda como son: la amplitud, la longitud de onda, el periodo y del calculo de su frecuencia y velocidad de propagación. 
El objetivo del presente trabajo se encuentra centrado en que el estudiante este en capacidad de identificar, representar y explicar la naturaleza y características de una onda, los fenómenos ondulatorios y el movimiento del medio en que se propaga una onda, utilizando un modelo virtual complementario de la experiencia real.

Esta investigación utiliza las nuevas tecnologías de la información y la comunicación como una herramienta o mediación instrumental para resolver una actividad (construcción del concepto de onda y de fenómenos ondulatorios), hecho que introduce cambios respecto a la motivación y a los procesos cognitivos de los estudiantes a quienes les agrada el manejo de la virtualidad y con quienes se puede llegar a construir otros conceptos utilizando modelos virtuales. Aquí las simulaciones, empleadas en los modelos virtuales, son consideradas instrumentos en el sentido atribuido por la psicología histórico-cultural a los auxiliares utilizados para resolver una actividad. En los trabajos de Vygotsky se plantea una estrecha relación entre actividad y mediación instrumental, a la que le atribuyen como resultado la estructura básica de la cognición (Vygotsky.1979:89 por PEREA, 1999).

\section{REFERENTES EPISTEMOLÓGICOS}

Los modelos virtuales establecen una estrecha relación con el modelo cognitivo (GIERE, 1992) debido a que resultan apropiados para ser aplicados en la Ciencia Escolar puesto que establecen una conexión entre los modelos teóricos y las representaciones mentales (modelos) que tienen los estudiantes acerca de los fenómenos naturales.

Dentro de los diferentes modelos de ciencia (el empirismo y neopositivismo; el racionalismo; el constructivismo), se toma el modelo cognitivo de ciencia, como base para fundar la construcción del concepto de onda y de fenómenos ondulatorios por medio de un modelo virtual, teniéndose en cuenta que el modelo cognitivo de ciencia muestra una estrecha relación entre los experimentos, los lenguajes y las teorías científicas y en segundo lugar porque permite fundamentar, epistemológicamente, de manera autónoma, a la Ciencia Escolar en su relación con los estudiantes (Giere, 1992, 1996; Izquierdo et al, 1996, 1999, citado en Perea 1999).

\section{REFERENTES PEDAGÓGICOS Y DIDÁCTICOS}

En el proceso de enseñanza el profesor debe actuar como orientador, facilitador o mediador del aprendizaje del estudiante, debe motivar, manifestar intencionalidad, provocar actividades dinámicas y situaciones en las que los estudiantes puedan construir, de construir y reconstruir (hacer aprendizaje significativo) sus propios 
conocimientos y valores. Las actividades que proponen el profesor y los materiales didácticos de apoyo que usa no cumplirán su auténtica misión, si el educador no manifiesta esta intencionalidad educativa. Según Vygotsky se deberá acudir a la didáctica y a la mediación instrumental para tener referentes propositivos que le permitan acortar la distancia entre las finalidades educativas y la realidad de sus alumnos, es decir, reducir la ZDP. Es por ello que en este trabajo se presente una concepción de la actividad del profesor, obtenida a la luz de la teoría de la actividad de Vygotsky aplicada por AlekséiNikoláyevichLeontiev.

Para Leontiev, en cualquier actividad humana el sujeto (el profesor), impulsado por sus motivos, actúa sobre el objeto (la dirección del aprendizaje del estudiante) para alcanzar su objetivo (la representación que ha imaginado del producto a lograr). El sujeto planifica la actividad, los procedimientos y los medios que va a utilizar, las condiciones en que se debe realizar y el producto a lograr. Los medios son los instrumentos materiales que posee el sujeto y que emplea en la transformación del objeto, en nuestro caso, el modelo virtual con sus simulaciones. Los productos son los resultados logrados mediante la actividad, en nuestro caso la construcción del concepto de onda y de fenómenos ondulatorios utilizando un modelo virtual.

\section{METODOLOGÍA}

La metodología desarrollada está de acuerdo a lo propuesto en la.Etnografía y diseño cualitativo en investigación educativa", (GOETZ y LeCOMPTE, 1988), en donde se aplica la propuesta didáctica, de manera sincrónica, a un grupo homogéneo y diferenciado de estudiantes de grado once, con edades entre los 15 y 17 años, de un colegio privado, coeducativo, tomando como referente el tema de las ondas que se estudia en el primer periodo académico.

Se establecieron las siguientes tres variables para hacerles seguimiento en el curso de la investigación:

I. Naturaleza y características de una onda.

II. Movimiento del medio en el cual se propagan las ondas

III. Fenómenos de onda: reflexión, refracción y difracción e interferencia.

Los estudiantes recibieron tres semanas de clases magistrales apoyadas por demostraciones dinámicas hechas con un resorte helicoidal y un laboratorio con la cubeta de ondas. Después de cubrir el tema de ondas, propuesto en la planeación periódica, se les aplicó a los estudiantes un cuestionario de diagnóstico para medir el grado de apropiación y manejo de las variables establecidas. Simultáneamente se aplicó una encuesta estructurada a profesores de educación media y superior para indagar sobre el uso de modelos y el uso de las TICs, como herramientas didácticas de apoyo en la enseñanza de la Física, ondas en particular. Luego se 
desarrolló con los estudiantes la propuesta didáctica como una mediación instrumental y finalmente se obtuvieron los resultados medidos por medio del análisis de un segundo cuestionario de control aplicado al final del estudio, en donde se evaluó el comportamiento y la evolución de las mismas variables.

\section{PROPUESTA}

Se eligieron dos plataformas que admiten descargar e instalar sus programas libremente en el computador y que permiten trabajar en línea desde su página Web (para su normal funcionamiento se necesita instalar en el computador el programa Java y el plug-in Descartes para Web 2.0.):

- Plataforma PhET, PhysicsEducationTechnology, desarrollada por University of Colorado. Funciona bajo el ambiente de todos los sistemas operativos (Windows, Mac OS X y Linux), con el programa Java instalado.

- Plataforma Proyecto Newton, desarrollada por el Instituto de Tecnologías Educativas (ITE) - Centro Nacional de Investigación y Comunicación Educativa (CNICE) -Ministerio de Educación,Gobierno de España.

Funciona bajo el ambiente de todos los sistemas operativos (Windows, Mac OS X y Linux), con el programa Java instalado y con el plug-in Descartes Web 2.0, que se ofrece libremente en la misma página. Las plataformas presentan los modelos en forma de simulaciones (applets) o herramientas interactivas que permiten a los estudiantes establecer conexiones entre los fenómenos de la vida real y la ciencia subyacente que explica esos fenómenos, usan las analogías para dar sentido a fenómenos desconocidos y sirven para cambiar las formas tradicionales de la participación de los estudiantes en el aula. Las simulaciones presentadas se desarrollan atendiendo a competencias semióticas y están orientadas para ayudar a los estudiantes a visibilizar conceptos científicos debido a que animan lo que es invisible para el ojo y que no son plausibles en el mundo real. Las plataformas presentan un menú que permite la manipulación de sus herramientas interactivas tales como gráficos, controles intuitivos, reguladores, botones de opción e instrumentos de medida (reglas métricas, cronómetros, y multímetros), por medio de los cuales los estudiantes ilustran las relaciones de causa - efecto. Los programas utilizados en las dos plataformas, están diseñados bajo los parámetros de una tecnología virtual, que pretenden introducir a los usuarios dentro de micro mundos digitales. Los micro mundos son un ejemplo de ambientes de aprendizaje activo, en donde los estudiantes deben participar e interactuar con el ambiente en que están inmersos, con el fin de crear su propia visión del tema. (David H. Jonassen, 2000, citado por SANTOS, G y Stipcich, S, 2009). 
- El primer paso es acceder a las siguientes páginas (o trabajar desde el programa instalado previamente en el computador): http://phet.colorado.edu/sims/wave-ona-string/wave-on-a-string_es.html.

El estudiante debe identificar, reconocer y manejar las herramientas que presenta el panel de control. Una vez conozca el funcionamiento del programa, el estudiante debe producir ondas viajeras (en el modo de oscilación, sin final y sin amortiguación en el panel de control), y por el método interactivo causa-efecto, debe explicar el significado de las características de las ondas y medir algunos de sus parámetros con sus correspondientes unidades de medida.

Los objetivos del laboratorio virtual son: Medir longitudes de onda _, Medir periodos $T$, Calcular frecuencias $f$, Calcular velocidad de onda v, Medir amplitudes A, Establecer la relación entre la frecuencia y la longitud de onda.

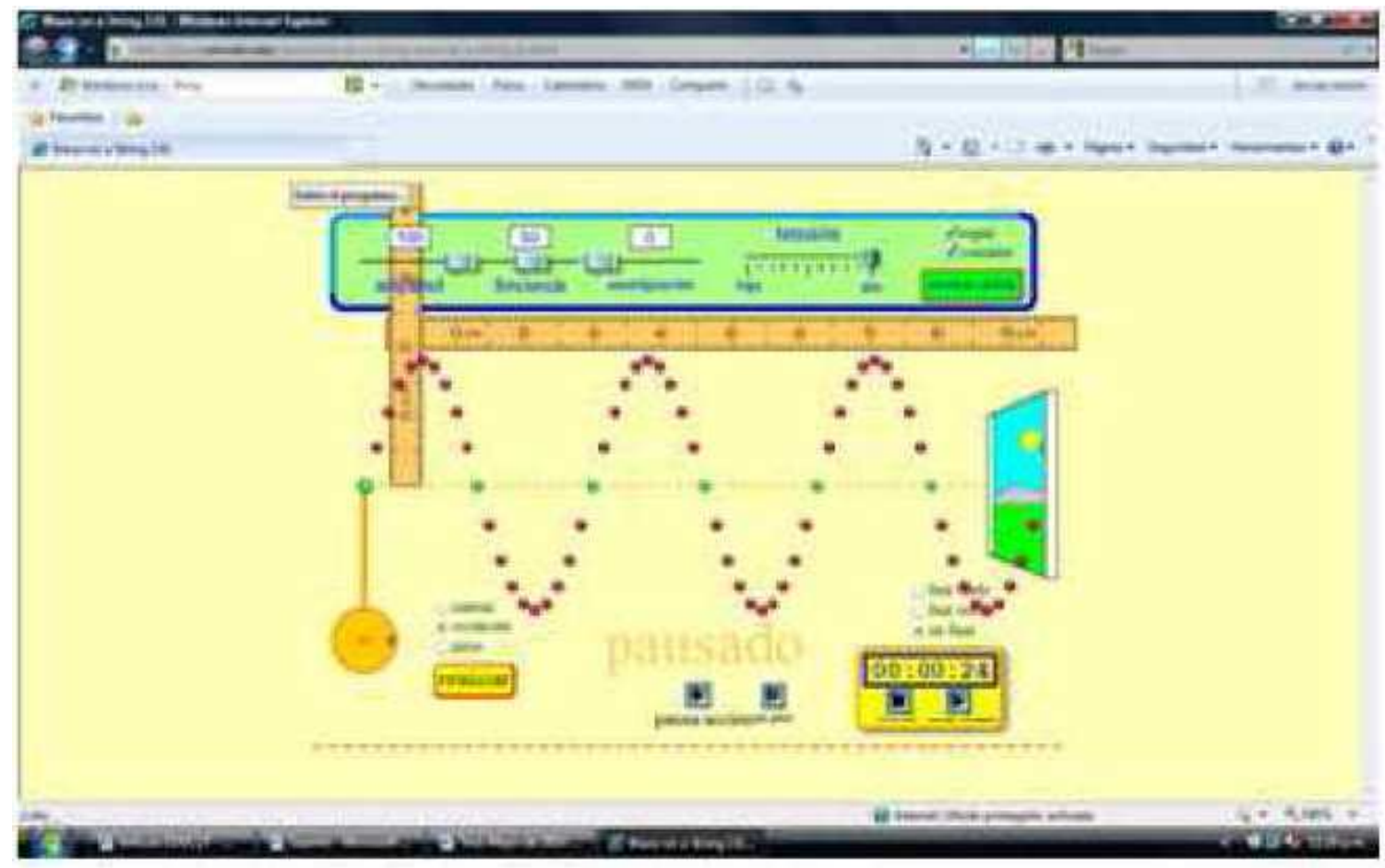

Figura 1. Práctica I.

Ingresar en la página (o trabajar desde el programa instalado previamente en el computador):

http://concurso.cnice.mec.es/cnice2005/56_ondas/index.htm.

El estudiante debe identificar, reconocer y manejar las herramientas quepresenta el panel de control. Una vez conozca el funcionamiento delprograma, el estudiante debe desarrollar el Laboratorio I. (, T, f, v, A), ayudado por una guía de trabajo, en donde se recomienda hacer unaanálisis de errores para cada una de las mediciones. 
Al finalizar el trabajo realizado en las dos páginas Web sugeridas, elestudiante debe producir un informe, en parejas, en donde consigne amodo de conclusiones el cumplimiento de los objetivos propuestos.

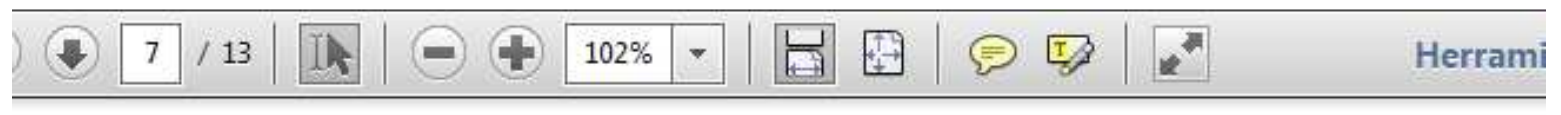

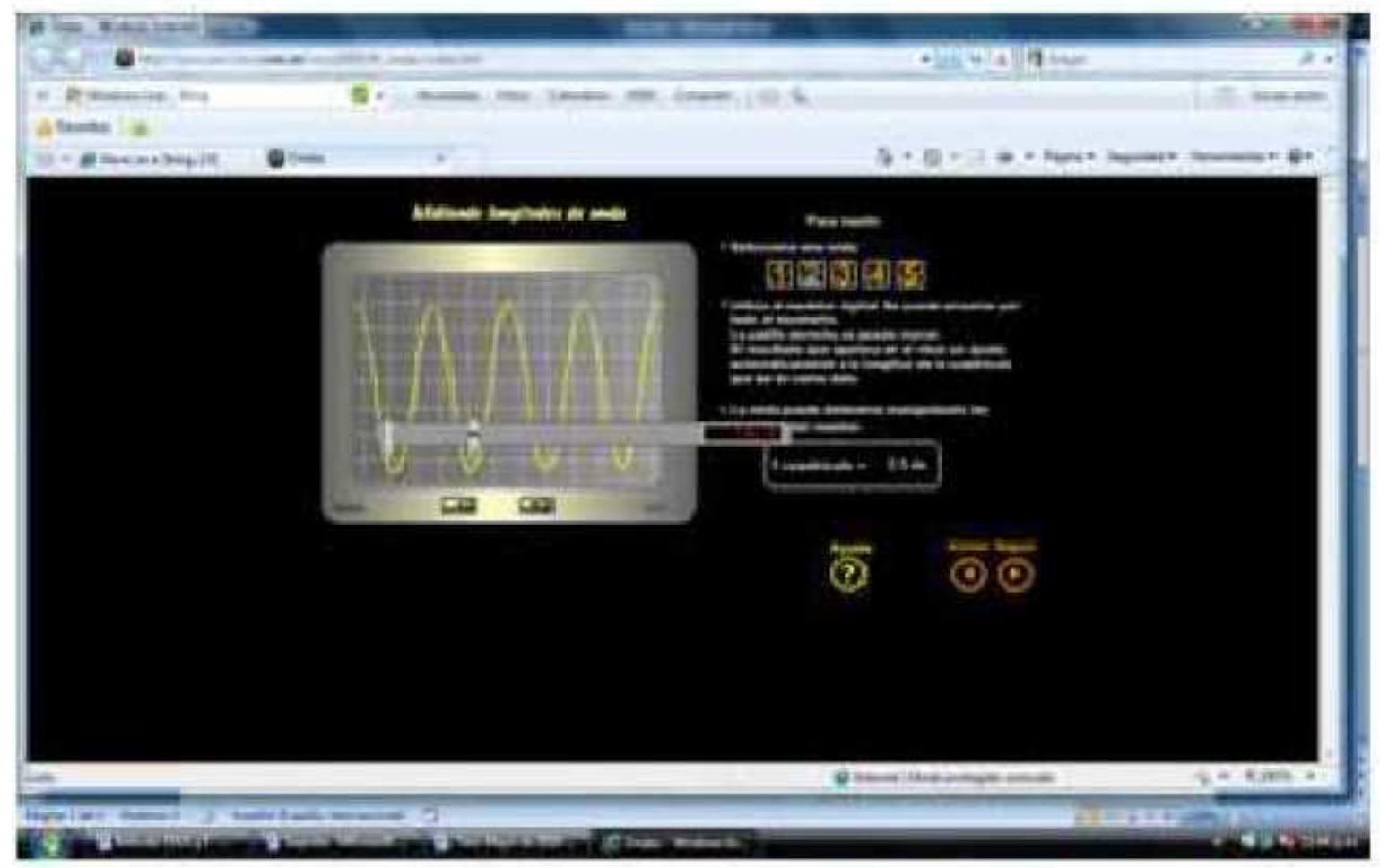

Figura 2. Práctica I y II.

- El segundo paso es acceder a las siguientes páginas (o trabajar desde el programa instalado previamente en el computador):

http://concurso.cnice.mec.es/cnice2005/56_ondas/index.htm.

El estudiante desarrolla el Laboratorio II (Reflexión) y posteriormente el Laboratorio III (Refracción), ayudado por una guía de trabajo.

http://recursostic.educacion.es/newton/web/materiales_didacticos/ondas/ ondas-interferencia1.htm?2|\&0.

El applet representa el fenómeno de la interferencia y se abre dando clic en avanzar. Se realizan las prácticas A1, A2 y A3 siguiendo las consignas que se indican para cada una de ellas.

http://recursostic.educacion.es/newton/web/materiales_didacticos/ondas/ 
ondas-difraccion1.htm?3\&1.

El applet representa la difracción de los frentes de onda y se abre dando clic en avanzar. Se realizan las prácticas $\mathrm{A} 1$ y $\mathrm{A} 2$, siguiendo las consignas que se indican para cada una de ellas.

Los objetivos del laboratorio virtual son:

Comprender identificar y explicar de manera cualitativa y cuantitativa los fenómenos ondulatorios y la comprobación de dos leyes fundamentales:

Reflexión, Refracción, Difracción, Interferencia, Comprobar la Ley de la reflexión y la ley de Snell.

Al finalizar el trabajo realizado en las tres páginas Web propuestas, el estudiante debe producir un informe, en parejas, en donde consigne a modo de conclusiones el cumplimiento de los objetivos propuestos.

Finalmente, para deducir la ecuación de onda se debe acceder a la página (o trabajar desde el programa instalado previamente en el computador): http://newton.cnice.mec.es/materiales didacticos/MAS/241 mas.html?1\&3.

El estudiante debe encontrar la semejanza entre el movimiento armónico simple, M.A.S y el movimiento circular uniforme, M.C.U y de allí debe obtener ecuación que describe dichos movimientos, conocida como la función de onda. 

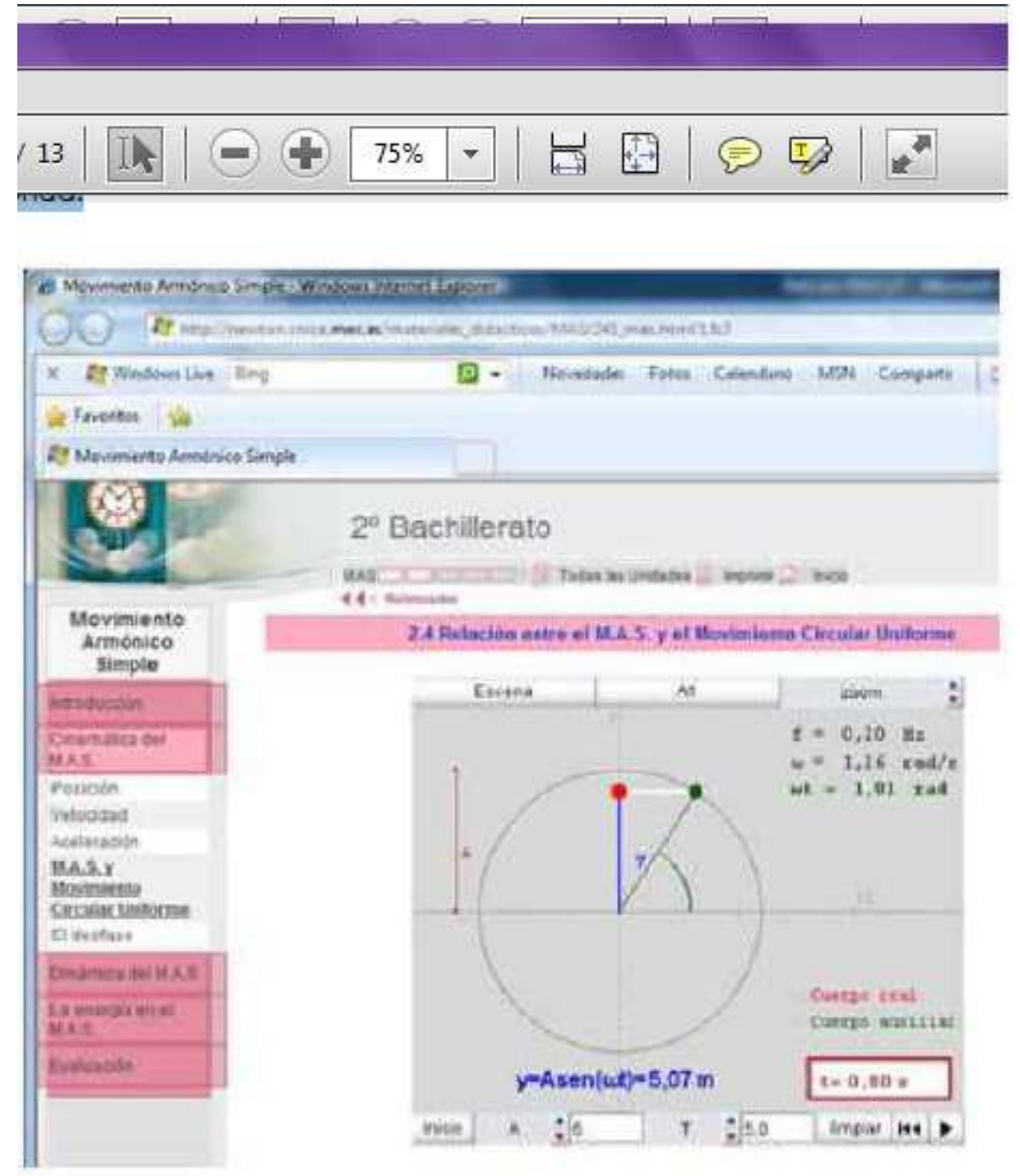

Figura 3. Semejanza entre M.A.S y M.C.U. 


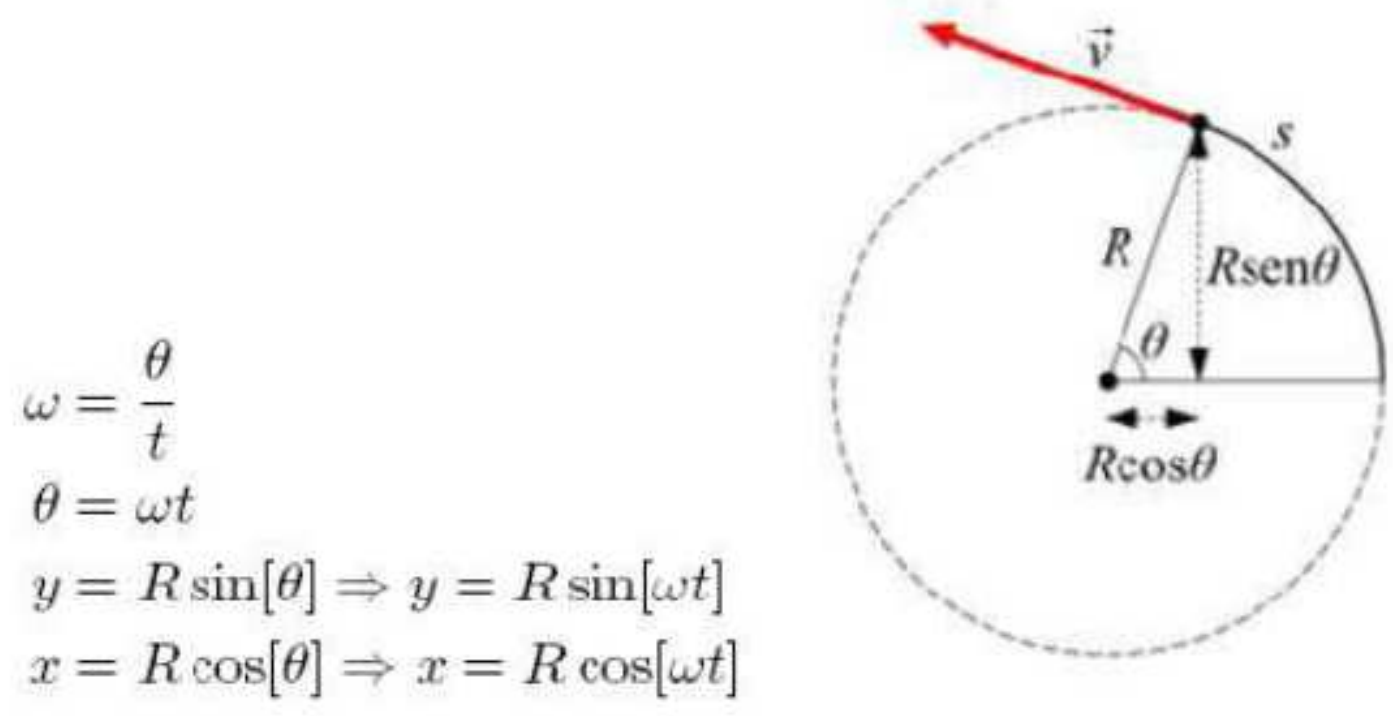

\section{RESULTADOS}

En la tabla1 y la figura 5 . Se muestra la evolución que presenta el conjunto de estudiantes, en la construcción de los conceptos contenidos en las variables analizadas en el curso de la investigación. Las tres variables estudiadas muestran un comportamiento satisfactorio en el curso del estudio.

Para el análisis de las variables se fijó una meta de aceptación del $60 \%$, equivalente a una calificación de 3.0 en la escala de 0 a 5.0. Al realizar el estudio, en el cuestionario de control, sobre el grado de interiorización de las tres variables estudiadas, se muestra una deficiencia en las dos primeras ya que no alcanzan el $60 \%$ que se propone como meta y la tercera variable, escasamente lo logra.

Los temas que contienen los conceptos en los cuales los estudiantes muestran tener mayor dificultad en su construcción e interiorización, son los referidos al reconocimiento de la existencia de las fuerzas de recuperación (le llaman onda a la ola producida en un estadio de futbol), a la explicación del movimiento del medio en que se propagan las ondas, especialmente con las ondas longitudinales, y a la identificación del fenómeno de la difracción. Estos tres temas se tratan con particular cuidado en el desarrollo de los laboratorios virtuales.

\section{CONCLUSIONES}

El uso de simulaciones, como instrumentos para resolver una actividad (construcción del concepto de onda y de fenómenos ondulatorios), favorece el aprendizaje dado que introduce cambios respecto a la motivación y a los procesos cognitivos de los estudiantes. Aquí, los applets son considerados instrumentos en el sentido atribuido por la psicología histórico-cultural a los auxiliares utilizados 
para resolver una actividad. Esta perspectiva brinda elementos teóricos y metodológicos para estudiar de manera holística el aprendizaje de las ciencias, ondas en particular, con tecnología informática como una actividad cognitiva, que bien podría considerarse, producto de una evolución filo y ontogenética, donde el desarrollo histórico cultural del hombre tiene un rol preponderante. En los trabajos de Vygotsky se plantea una estrecha relación entre actividad y mediación instrumental (como muestra el mapa conceptual a continuación), a la que le atribuyen como resultado la estructura básica de la cognición. Habida cuenta de identificar dos tipos de actividad, una orientada externamente y la otra orientada internamente, se distingue entre "herramienta como medio para el trabajo y lenguaje como medio para el intercambio social"(Vygotsky.1979:89). De manera que los sistemas de herramientas y los sistemas de signos no pueden considerarse isomorfos (con la misma estructura), dado que orientan de distinto modo la actividad humana, sino instrumentos que comparten la característica de tener una función mediadora (SANTOS, G y Stipcich, S, 2009)

Los modelos virtuales (applets o simulaciones por computador) se consideraninstrumentos que favorecen la construcción de conocimientos, expanden lasposibilidades de manipular y transformar los objetos, promueven el desarrollode funciones psicológicas superiores para interpretar, organizar, planificar,comparar, relatar, seleccionar, tomar decisiones y representar elconocimiento empleando diferentes lenguajes que comprometencompetencias cognitivas diversas (SANTOS, G y Stipcich, S, 2009). Interactuarcon una simulación, con los pares de la clase, con el docente y con el saberteniendo como finalidad dar respuesta a una actividad implica afrontar unavariedad de lenguajes tales como: el tecnológico, el cotidiano queempelará para discutir con sus compañeros y con el docente, el científico(que incluye los términos disciplinares específicos), el algebraico (de lasecuaciones que se pongan en juego) y el gráfico.

El mapa conceptual, mostrado en la figura 5, muestra las funciones de losinstrumentos psicológicos. Se inicia con el concepto de instrumento porqueVygotski parte de la analogía de los instrumentos materiales, que transformanla naturaleza $y$, por tanto, median la actividad del hombre en los procesosde transformación de ella, y los instrumentos psicológicos, que sirven paraactuar internamente y no externamente, controlando y transformando losprocesos.

De lo anterior precisamos las conclusiones así:

- La metodología utilizada en la investigación es apropiada, debido a queella conduce a obtener resultados que permiten hacer una propuesta paramejorar la construcción del concepto de onda y de fenómenos ondulatorios. 
- Combinar los dos análisis cualitativo y cuantitativo en la investigaciónmuestra ser altamente productivo, puesto que siendo métodoscomplementarios, permiten dilucidar variables que de otra manerapermanecerían ocultas.

- Los estudiantes construyen el concepto de onda a partir de un modeloestático que no les permite visualizar la componente dinámica de las ondasy tampoco interpretar la ecuación que sirve para describir el movimientoondulatorio: La Función de Onda.

- El utilizar un modelo estático de onda no les permite a los estudiantesidentificar sus características (amplitud, $v$ longitud de onda) ni medir losparámetros dinámicos (periodo, velocidad de propagación, cálculo de lafrecuencia e identificación de las fuerzas de recuperación), por lo tanto, lesdificulta explicar el movimiento del medio en que se propagan las ondas, particularmente el asociado a las ondas longitudinales (un alto porcentajeno identifica la difracción del sonido).

- El 10\% de los estudiantes que participan en el estudio no muestran interéspor la propuesta didáctica ni tampoco un avance significativo en laconstrucción de sus conceptos sobre ondas y fenómenos ondulatorios.

\section{REFERENCIAS}

[1] AD'URIZ-BRAVO, Agustín, G'OMEZ, Adrianna, M`ARQUEZ, Conxita,SANMART'I, Neus. La mediaci'on analógica en la ciencia escolar. Propuestade la "función modelo teórico". Universidad de Buenos Aires UniversidadAutónoma de Barcelona, Bellaterra, Espa na.2005.

[2] AGUILAR, T Manuel Francisco, El mapa conceptual y la teoríasociocultural, Universidad Autónoma del Estado de Morelos, M'exico,2006.

[3] ARRUDA, José Ricardo. Un Modelo Didáctico para Enseñanza Aprendizajede la Física. Instituto de Física, Universidad de Estado do Rio de Janeiro.Revista Brasileira de Ensino de Física, vol. 25, no. 1, Marzo, 2003.

[4] ANDR'ES, Ma. Maite, PESA, Marta, MENESES, Jesús. Desarrollo conceptualacerca de ondas mecánicas en un laboratorio guiado por el modeloMATLaF. Caracas Venezuela, Tucumán Argentina, Burgos España. RevistaElectrónica de Enseñanza de las Ciencias Vol. 5No 2 (2006).

[5] CHAPARRO CHAVES, Elisa Johanna, GUERRERO, Jobana López, VILLALBACAMPOS Mónica, GARC'IA MART'INEZ, Álvaro. Representaciones epistémicocognitivas del concepto 'acido-base. Universidad Distrital Francisco José deCaldas. Bogotá. IIEC, Volumen 1, No. 1, 2006: 60- 68.2006. 
[6] CHIAPPE LAVERDE, Andrés. Acerca de lo pedagógico en los objetos deaprendizaje-reflexiones conceptuales hacia la construcción de su estructurateórica. Universidad de la Sabana. Bogotá. Estudios Pedagógicos XXXV, No 1:261-272. 2009.

[7] D 'AVILA ESPINOSA, Sergio. El papel del profesor ante el aprendizaje y laenseñanza. Universidad Autónoma de San Luis Potosí. México 1999. NuevaAlejandría, el Portal de los Educadores, Disponible en:http://www.nuevaalejandria.com/akademeia/sdavila/aprense.html

[8] D 'AVILA ESPINOSA, Sergio El aprendizaje significativo. Esa extrañaexpresión. (utilizada por todos y comprendida por pocos), UniversidadAutónoma de San Luis Potosí, México 2000. Disponible en:http://depa.pquim.unam.mx/amyd/archivero/AUSUBELAPRENDIZAJESIGNIFIC ATIVO_1677.pdf

[9] GARC'IA, Luis Ignacio, 2004. .Ondas". Centro Nacional de Investigación yComunicación Educativa (CNICE) - Ministerio de Educación, Portal de laConsejería de Educación y Ciencia del Gobierno del Principado de AsturiasEspaña, disponible en:http://concurso.cnice.mec.es/cnice2005/56 ondas/index.htm

[10] GIERE, Ronald N. La explicación de la ciencia. Un acercamiento cognoscitivo. Editorial: Concejo Nacional de ciencia y tecnología,México. 1992.

[11] GOETZ, Judith-LeCOMPTE, Margaret. Etnografía y diseño cualitativo eninvestigación educativa. Editorial: Morata. Madrid. 1988.

[12] GOBIERNO DE ESPA NA, Ministerio de Educación, Instituto de Tecnologías Educativas (ITE), Centro Nacional de Investigación y ComunicaciónEducativa (CNICE). Proyecto Newton, taller abierto de creación de recursosinteractivos para la enseñanza de la Física. España. 2010.

[13] ICFES, Examen de estado de la calidad de la educación superior,pruebas realizadas en los años 2003 a 2006.

[14] MANCINI, Luis Leonardo - MARTINEZ, María Sandra. Nuevos enfoquessobre la enseñanza. Estrategias para una práctica pedagógica eficaz.Primera edición. Editorial: Ediciones Santillana S.A. 2004.

[15] MINISTERIO DE EDUCACI'ON NACIONAL, República de Colombia.Estándares Básicos de Competencias en Ciencias Naturales y CienciasSociales. Serie guías No 7. 2004. 
[16] MIRANDA, Andrea, Análisis semiótico en el diseño de simulaciones paraaprender ciencias. Una perspectiva desde la tríada de Peirce. RevistaElectrónica Razón y Palabra, ISSN 1605-4806, No. 63, México, 2008.

[17] PEREA, Álvaro. Razonamientos de estudiantes de secundaria sobre los procesos de cambio". Tesis Doctoral. Directora: Mercé Izquierdo i

Aymerich.Universidad Autónoma de Barcelona. Bellaterra, Barcelona.1999.

[18] SANTOS, Graciela, STIPCICH Silvia. Múltiples representaciones en losapplets: una alternativa para la apropiación de los códigos básicos enciencia y tecnología. Revista Electrónica Razón y Palabra, ISSN 1605-4806, No.69, 2009 México. 2009.

[19] UNIVERSIDAD DE COLORADO, Physics Education Technology

(PhET)University of Colorado at Boulder, Interactive ScienceSimulations, Sound \& Waves, http://phet.colorado.edu/simulations/. 2010.

[20] VIAFARA ORTIZ, Robinson. La relación entre la educación en ciencias y las NITC a través del diseño, desarrollo y aplicación de un programaeducativo multimedia. Universidad del Valle, Educación en Ciencias yTecnología. El Hombre y la Máquina No 26. Universidad Autónoma deOccidente. 2006.

[21] VIDAL CASTA NO, Gonzalo. La actividad del profesor. Universidad de laHabana. Universidad Autónoma de Nuevo León, México. UniversidadAutónoma del estado de México. 2005. Disponible en:http://www.educar.org/articulos.

[22] ZAMORA B, Jesús P. El naturalismo científico de Ronald Giere y PhilipKitcher. Un ensayo de comparación critica. Universidad Carlos III, Madrid.2000.

\section{CIBERGRAFÍA}

1. http://phet.colorado.edu/sims/wave-on-a-string/wave-on-a-string_es.html

2. http://newton.cnice.mec.es/newton2/Newton_pre/index.html

3.http://newton.cnice.mec.es/materiales_didacticos/MAS/241_mas.html?1\&3

4. http://concurso.cnice.mec.es/cnice2005/56_ondas/index.htm

5. http://recursostic.educacion.es/newton/web/materiales_didacticos/ ondas/ondas-transversales1.htm?1\&0

6. http://recursostic.educacion.es/newton/web/materiales_didacticos/ ondas/ondas-longitudinales $1 . h t m ? 1 \& 1$

7.http://recursostic.educacion.es/newton/web/materiales_didacticos/ondas2/ondas -objetivos.html 